Copyright: ( 2016 IEEE. Personal use of this material is permitted. Permission from IEEE must be obtained for all other uses, in any current or future media, including reprinting/republishing this material for advertising or promotional purposes, creating new collective works, for resale or redistribution to servers or lists, or reuse of any copyrighted component of this work in other works.

\title{
Gamification in crowdsourcing: A review
}

\author{
Benedikt Morschheuser \\ $\mathrm{CR}$, Robert Bosch GmbH and \\ Karlsruhe Institute of Technology \\ benedikt.morschheuser@, \\ de.bosch.com
}

\author{
Juho Hamari \\ Game Research Lab \\ School of Information Sciences \\ University of Tampere \\ juho.hamari@uta.fi
}

\author{
Jonna Koivisto \\ Game Research Lab \\ School of Information Sciences \\ University of Tampere \\ jonna.koivisto@uta.fi
}

\begin{abstract}
This study investigates how different gamification implementations can increase crowdsourcees' motivation and participation in crowdsourcing (CS). To this end, we review empirical literature that has investigated the use of gamification in crowdsourcing settings. Overall, the results of the review indicate that gamification has been an effective approach for increasing crowdsourcing participation. When comparing crowdcreating, -solving, -processing and rating CS approaches, the results show differences in the use of gamification across CS types. Crowdsourcing initiatives that provide more monotonous tasks most commonly used mere points and other simpler gamification implementations, whereas CS initiatives that seek for diverse and creative contributions have employed gamification in more manifold ways employing a richer set of mechanics. These findings provide insights for designers of gamified systems and further research on the topics of gamification and crowdsourcing.
\end{abstract}

\section{Introduction}

During recent years modern ICT technologies have spawned two interwoven phenomena: gamification and crowdsourcing (CS). Today, multitude of different organizations employ crowdsourcing (CS) as a way to outsource various tasks to be carried out by 'the crowd'; a mass of people reachable through the internet (see [24]). The rapid diffusion of these technologies can be seen both in industry as well as in the academia [13, 24]. Business analysts have estimated that a majority or at least $50 \%$ of organizations have gamified some of their processes by $2015[14,26]$. As illustrated in Figure 1, the body of literature on both CS and gamification has been rapidly growing. Moreover, these technologies appear together frequently: crowdsourcing is one of the major application areas for gamification [20]. Naturally, the main goals of CS in general are either cost savings or the possibility to innovate solutions that would be difficult to cultivate in-house. However, CS relies on the existence of a reserve of people that are willing to take on tasks for free or for a small monetary compensation. Along this reasoning, CS tasks are increasingly gamified, that is, organizations attempt to make the work activity more like playing a game in order to provide other motives for working than just the monetary compensation.

However, while the union of these novel technological phenomena seems intuitively appealing, there has still been a dearth of coherent understanding of the use of gamification in CS. Although singular scattered empirical pieces on the topic exist, efforts have not yet been made to collate and synthesize this body of knowledge. Moreover, both CS and gamification can take a variety of forms and it would be short-sighted to assume that differing gamification implementations would function similarly across different CS approaches.

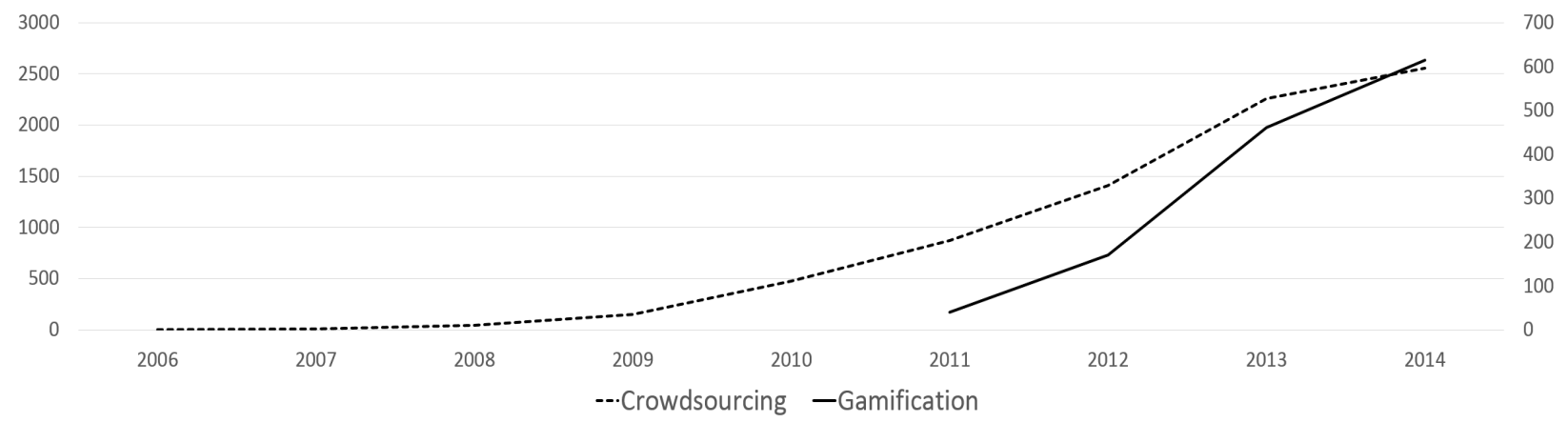

Figure 1. Search hits (Scopus, all fields, CS left axis, gamification right axis) 
Therefore, in this paper we conduct a review of studies that have investigated the use of differing gamification implementations across different types of crowdsourcing initiatives. We review the results reported in the analyzed literature, the research methods used and the investigated motivations. Furthermore, we examine how gamification has been implemented to provide insights for designers of gamified crowdsourcing systems.

\section{Background}

Crowdsourcing refers to outsourcing work, tasks or problem solving to people online rather than to employees or traditional suppliers [13, 24]. It has been considered to be a particularly useful way to coordinate work for tasks that can benefit from a collective intelligence [37] or that are difficult to process by computers and are therefore outsourced to humans (also see "human computation" [1]).

Based on Geiger \& Schader [15], crowdsourcing can broadly be categorized into four categories (See Figure 2). First, crowdsolving approaches use the diversity of the crowd to find a huge number of heterogeneous solutions to a given problem. The value of this approach results directly from each isolated contribution (non-emergent). Crowdsolving is often used for very complex problems (e.g. Foldit, a game based approach to optimize protein folding - see [6]) or if no pre-definable solution exists (e.g. ideation contests). Second, crowdcreation solutions aim to create comprehensive (emergent) artefacts based on a variety of heterogeneous contributions. Typical examples include all kinds of user-generated content (e.g. Youtube) or knowledge derived from collaborative aggregation (e.g. Wikipedia). Third, crowdrating systems commonly attempt to harness the so-called "wisdom of crowds" [51] to perform collective assessments or predictions. In this case, the emergent value arises from a huge number of homogeneous "votes" (e.g. NASA Clickworkers, at which the clicks/votes of a crowd were used to identify craters on asteroids [31]). Fourth, crowdprocessing approaches rely on the crowd to perform large quantities of homogeneous tasks. Identical contributions are a quality attribute for the validity of the work. The value is derived directly from each isolated contribution (non-emergent) (e.g. Mechanical Turk or Galaxy Zoo [38]).

Since an active crowd of participants is crucial for successfulness of crowdsourcing, the motivation of the crowdsourcees is of great importance. Although a relatively large amount of research has been done in the area of crowdsourcing, only a small portion of studies actually investigates participants' motivation [57]. Existing studies have showed that there are several reasons for people to participate in crowdsourcing and related online work, ranging from intrinsic to extrinsic motivations [21, 32, 50, 58, 59]. For example intrinsic motivation, created by tasks that allow the participant to be creative and experience autonomy, to develop own skills and feel competent, to enjoy pastime or to achieve social recognition, can in some cases dominate extrinsic motivation evoked by financial payoffs or external social reasons [32]. Further, task characteristics [32, 59], task granularity [58] or perceived motivational affordances [58] can have an influence on the individual's motivation.

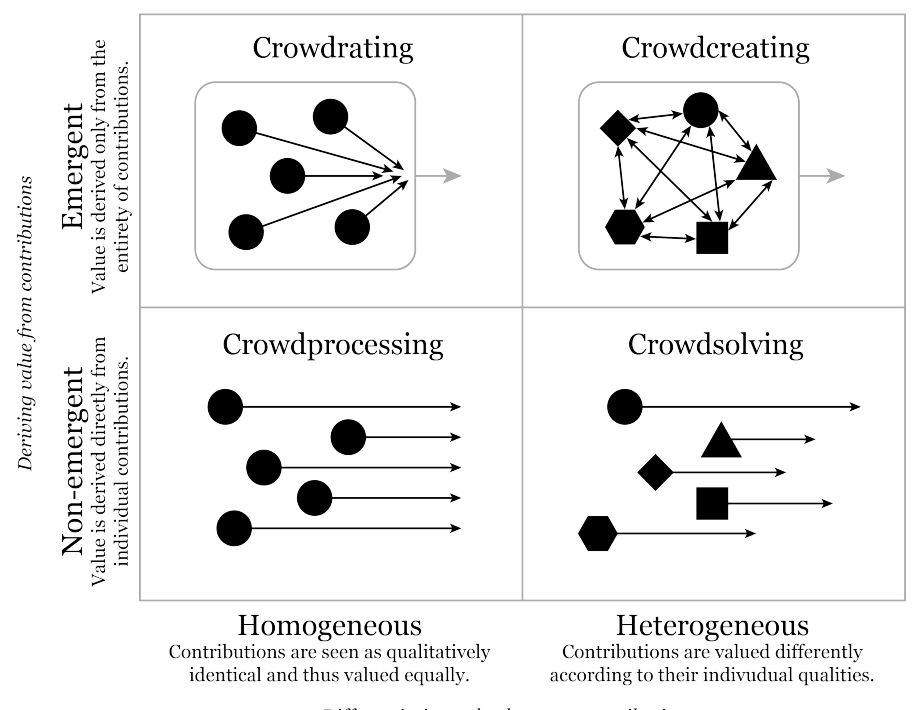

Differentiating value between contributions

Figure 2. Four archetypes of crowdsourcing systems based on Geiger \& Schader [15] 
Therefore, one major challenge in motivating people to participate is to design a crowdsourcing system that promotes and enables the formation of positive motivations towards CS work as well as fits the type of the activity. For instance, whereas some crowdsourcing approaches aim for systematically derived contributions, other crowdsourcing types may call for incentive structures that promote creativity. In other words, as the CS activities can differ dramatically, so can the means by which to motivate crowdsourcees in a given CS initiative.

In the area of incentive design in information system field, one of the most popular developments in recent years has commonly been titled as gamification $[18,20]$. Gamification refers to design that attempts to, firstly, increase the intrinsic motivation of users or participants to engage in a given activity or behavior and, secondly, to increase or otherwise change the given behavior. The term of gamification stems from the notion that games if anything are a pinnacle form of hedonic self-purposeful systems [19]. Most gamification applications borrow design patterns from (video) games and, consequently, aim to give rise to similar experiences as games commonly do, e.g: feelings of mastery, autonomy, flow, suspense etc. (see e.g. [25]). If we consider gamification in the context of $\mathrm{CS}$, gamification can be seen as an attempt to redirect crowdsourcees' motivations from purely rational gainseeking to self-purposeful, intrinsically motivated activity: "Transforming Homo Economicus into Homo Ludens" [17]. In other words, elements known from games act as motivational affordances $[25,29,56]$ for the intrinsic motivations. Points, badges, leaderboards, avatars, and stories are some of the most often used motivational affordances in gamification [20]. Previous literature has conceptualized gamification into a few main aspects: 1) the design (affordances), 2) the psychological mediators/outcomes of gamification, and 3) the (behavioral) outcomes of gamification [25]. Existing empirical works also suggest that contextual factors [17] and factors related to user have an effect [34].

\section{Literature review process}

Following the guidelines of Webster \& Watson [54] and Ellis [11], the analysis procedure started by a literature search. We decided to use Scopus database as our source of data, as it is the largest abstract and citation database of scholarly literature [12]. Scopus includes, for example, the AIS, ACM, IEEE and Science Direct libraries among many others.
As in this study we are particularly focusing on the use of gamification in crowdsourcing, the literature search in the Scopus database was conducted using the search query TITLE-ABS-KEY(GAMIF* AND CROWD*). The search resulted in all Scopus entries that include any permutation of the terms gamification and crowdsourcing in the entry metadata (title, abstract or keywords). We intentionally limited the search to the metadata since searching for the terms in all the text would result in a relatively large amount of false positives as many papers refer to gamification and/or crowdsourcing in passing. The search procedure was undertaken in March 2015.

The search query resulted in 50 hits. These 50 papers were then screened for inclusion and relevance using the following criteria: 1) The full paper can be acquired, 2) paper is in English (and has been published on an international venue), 3) the paper is about gamification and crowdsourcing instead of the terms just being mentioned in the metadata, 4) the paper is not a duplicate reporting the same study in several papers, and 5) the paper contains empirically derived results.

Of the initial 50 hits, one paper was excluded due to the full paper not being available, and one paper due to not being in English. Furthermore, four papers were excluded from the review due to criterion 3). Moreover, one duplicate was found. [39] and [40] describe the same experiment and report similar results. Therefore, we have merged the information of the two papers and handle it in the analyses as one entity ([40]). Also [42] and [46] analyze the same case, but these papers were not considered duplicates due to the fact that different data was gathered, differing methods were used and consequently, different results were reported. Finally, 28 papers matching the criteria were identified for the review.

In the second step (see [54]) of the literature analysis, the included papers were coded. Two researchers carried out this process independently. After coding the two individual sheets were compared, discussed and combined. Information of all the papers pertaining to A) crowdsourcing (crowdsourcing type (see [15]) and financial incentives), B) gamification (affordances, psychological mediators/outcomes, behavioral outcomes (see [20]) and scoring rules) and C) results of empirical studies were gathered.

In the third step, the 28 empirical papers included in the review were further categorized as either containing results regarding the effects of gamification or not containing any results about the effects of gamification. In the latter case, the papers simply described a gamified crowdsourcing implementation. 


\section{Results}

\subsection{How gamification is used?}

The reviewed body of literature employed 11 types of gamification affordances ${ }^{1}$, which indicates that gamification is used in a variety of ways in CS (see Table 1). However, points (in 22 cases) and leaderboards (in 20 cases) were clearly the most implemented gamification mechanics. Commonly, these two affordances were combined to create competition between the participants. Understandably, our results indicate that points and scores are employed in CS contexts where the task is more easily enumerable such as crowdprocessing and crowdrating, and which strive for a large number of homogenous contributions. The richest employment of gamification with the largest variety of affordances can be found in solving-related CS work, whereas crowdprocessing and crowdrating are more focused on simpler forms of gamification such as points and leaderboards. CS types of crowdcreating and crowdsolving differ from crowdrating and crowdprocessing in that the participation depends on a variety of heterogeneous contributions. Crowdsourcing related to creative and diverse contributions therefore might benefit from more manifold gamification solutions.

CS types of creating and rating differ from solving and processing in that the end-goal of the crowdsourced work is an emergent value from the collective of contributions. Therefore, it could be assumed that designers of gamified CS systems with emergent outcomes would rather use cooperative gamification designs compared to designs of nonemergent approaches. When analyzing the affordances used between these types, no notable differences could be found however. Competition-based designs with points and leaderboards that encourage individual work rather than cooperative work were used very often in crowdprocessing, solving and rating approaches. However, the scoring approaches differed based upon how points were awarded and from which actions they could be earned. In crowdprocessing approaches, where the sheer number of contributions is more important than quality [15], users were commonly rewarded from general participation (e.g. number of completed tasks [28], number of correct answers [27],

\footnotetext{
${ }^{1}$ For the analysis how gamification is used in CS, we collected and categorized the affordances mentioned in the reviewed studies. It is noteworthy that we did not evaluate how a certain affordance was implemented in any given study but instead relied on what was reported in the reviewed papers and categorized the elements based on the information provided by the authors. Neither did we compare the affordances reported across the studies. Therefore, variance is bound to exist within the reported affordance categories.
}

or number of visited locations [52]). Whereas in crowdrating approaches, where the output is more emergent, users were rewarded from the quality of the contributions (e.g. from quality of contribution rated by others [9], similarity/agreement with contributions of other crowdsourcees [10, 16, 22, 47]). In crowdsolving approaches both forms occurred equally (e.g. number of completed tasks [40,55], quality of contribution rated by others $[35,53])$. Unfortunately, the small amount of studies investigating gamification in the crowdcreation approaches limits the identification of a clear pattern in their gamification implementations.

In addition to different kinds of point awarding logics, the points and scoring affordances were combined with further elements in diverse ways across implementations; they were used in combination with for instance, time limits (e.g. [22, 30]), they were used as a basis for calculating the level of crowdsourcees (e.g. [36, 47]), with the ability to compare them between peers and teams (e.g. [36, 47]) as well as with badges and missions to visualize specific goals (e.g. [2, $35,42,46,53])$.

In most of the studies the incentive system was solely based on gamification (Table 1). Some studies additionally employ financial rewards, e.g. a small monetary compensation or a prize for the leaders on a high score list, to motivate the participants. Although studies suggest that extrinsic rewards (such as money) can potentially crowd-out intrinsic motivation ([7, 8]), [42] and [46] found in their experiment that gamification in combination with financial rewards can in fact increase the participation when compared to gamification alone. However, the authors investigated this only in the short-term and indicated that financial rewards in comparison to gamification may reduce the participation in the long-term.

Table 1: Incentive orchestration

\begin{tabular}{|l|l|l|}
\hline Incentive & Literature & $\#$ \\
\hline gamification & $\begin{array}{l}{[\mathbf{2}]^{1},[4],[\mathbf{9}],[\mathbf{1 6}],[23],} \\
{[\mathbf{2 7}]^{*},[\mathbf{2 8}],[30],[\mathbf{3 5}],} \\
{[\mathbf{3 6}],[40],[41],[43],[\mathbf{4 4}],} \\
{[\mathbf{4 5}],[\mathbf{4 7}],[48],[49],[52],} \\
{[\mathbf{5 3}],[55],\left([\mathbf{4 2}]^{*},[\mathbf{4 6}]^{*}\right)}\end{array}$ & \\
\hline $\begin{array}{l}\text { gamification + } \\
\text { financial rewards }\end{array}$ & $\begin{array}{l}{[3],[\mathbf{5}],[\mathbf{1 0}],[22],[\mathbf{3 3}],} \\
{[\mathbf{4 2}]^{*},[\mathbf{4 6}]^{*},\left([\mathbf{2 7}]^{*}\right)}\end{array}$ & 7 \\
\hline $\begin{array}{l}{ }^{1} \text { References in bold refer to studies in which empirical } \\
\text { results about gamification have been reported. } \\
\text { * as experimental condition }\end{array}$ \\
\hline
\end{tabular}


Table 2: Gamification affordances per CS type

\begin{tabular}{|c|c|c|c|c|c|}
\hline $\begin{array}{l}\text { Crowdsourcing } \\
\text { Type / affordances }\end{array}$ & $\begin{array}{l}\text { Processing } \\
N=7\end{array}$ & $\begin{array}{l}\text { Rating } \\
N=10\end{array}$ & $\begin{array}{l}\text { Solving } \\
N=8\end{array}$ & $\begin{array}{l}\text { Creating } \\
N=3\end{array}$ & $\begin{array}{l}\text { Frequency } \\
\text { Total }=\mathbf{2 8}\end{array}$ \\
\hline points / score & $\begin{array}{l}{[3],[27],[33]} \\
{[36],[44],[52]}\end{array}$ & $\begin{array}{l}{[\mathbf{9}],[\mathbf{1 0}],[\mathbf{1 6}],} \\
{[22],[30],[41],} \\
{[\mathbf{4 2}],[\mathbf{4 6}],[\mathbf{4 7}]}\end{array}$ & $\begin{array}{l}{[23],[\mathbf{3 5}]^{1},[40],} \\
{[\mathbf{5 3}],[55]}\end{array}$ & [45], [49] & 22 \\
\hline $\begin{array}{l}\text { leaderboards/ } \\
\text { rankings }\end{array}$ & $\begin{array}{l}{[3],[27],[28],} \\
{[33],[36],[52]}\end{array}$ & $\begin{array}{l}{[4],[\mathbf{9}],[\mathbf{1 0}],} \\
{[\mathbf{1 6}],[22],[42],} \\
{[46],[47]}\end{array}$ & $\begin{array}{l}{[23],[\mathbf{3 5}],[40],} \\
{[\mathbf{5 3}],[55]}\end{array}$ & {$[2]$} & 20 \\
\hline $\begin{array}{l}\text { badges/ } \\
\text { achievements }\end{array}$ & {$[\mathbf{2 8}],[\mathbf{3 6}],[52]$} & {$[41],[42],[46]$} & {$[40],[53]$} & [2], [49] & 10 \\
\hline levels & {$[3],[36]$} & {$[9],[47]$} & {$[43],[55]$} & {$[49]$} & 7 \\
\hline progress & {$[28],[36]$} & & {$[\mathbf{3 5}],[43],[\mathbf{5 3}]$} & & 5 \\
\hline feedback & {$[3],[27]$} & & {$[35],[40]$} & & 4 \\
\hline rewards & & {$[42],[46]$} & {$[5]$} & & 3 \\
\hline storytelling & {$[44]$} & & {$[48]$} & {$[49]$} & 3 \\
\hline missions & & & {$[35],[48]$} & & 2 \\
\hline virtual territories & & & {$[40]$} & {$[49]$} & 2 \\
\hline
\end{tabular}

Furthermore, [27] indicates that the output quality of paid CS can be worse. Considering how gamification is implemented in CS (see Table 2), it seems that monetary rewards have been used in implementations with simple gamification designs, mainly together with points and leaderboards.

\subsection{Does gamification work?}

All of the empirical studies on the effectiveness of gamification in CS report that gamification has a positive impact on CS work (Table 3). Most studies that directly compared a gamified and non-gamified approach (e.g. $[10,16,28,33,36,40,44,53])$ report several positive effects, like the increase of (long-term [36]) engagement $[10,28,33,36,44,53]$, quality of the output $[10,16,36]$ and reduction in cheating compared to traditional paid CS [10]. However, gamification does not necessarily lead to an increase in participation. One study measured very small differences compared to a control group without gamification ([42]). In addition to the above studies that employed direct comparisons, five studies reported positive results based on the users' perception of the gamified crowdsourcing system $[2,9,35,47]$ or based on the measured user engagement [45]. These, mostly descriptively reported results, show no effects of gamification per se, but can be seen as positive indicators for the acceptance of gamification in context of CS.

Nearly all of the analyzed papers measure the effectiveness of the gamified system by measuring behavioral outcomes such as participation or willingness to contribute as the dependent variable. In all empirical studies the quality or quantity of the dependent variables were measured by collecting log data or conducting a survey. Several studies were also analyzing psychological outcomes. Table 4 gives an overview to the literature in which results about psychological outcomes were reported. The psychological outcomes were not commonly measured using comprehensive measurement instruments; instead, they were mostly examined by simple questionnaires, qualitative observations, or the observations of how participants behaved was used as a proxy for psychological aspects. Currently, not a single study has used validated psychometric measurement instruments. Only one study ([5]) seemed to have used a validated measurement construct for the experience of fun. 
Table 3: The results of gamification on crowdsourced work in different types of studies

\begin{tabular}{|c|l|l|l|l|}
\hline Results & $\begin{array}{l}\text { Positive compared to } \\
\text { non-gamified approach }\end{array}$ & $\begin{array}{l}\text { Perceived as } \\
\text { positive }\end{array}$ & Design studies & Frequency \\
\hline Quantitative & & & & \\
\hline$-\quad$ inferential & {$[10],[36],[44]$} & & {$[5],[27],([36])$} & 5 \\
\hline$-\quad$ descriptive & {$[40]$} & {$[45]$} & & 2 \\
\hline Qualitative & & {$[47]$} & {$[46]$} & 2 \\
\hline Mixed methods & & & & \\
\hline$-\quad$ inferential & {$[28],[33],[53]$} & {$[2]$} & {$[42],([28])$} & 5 \\
\hline$-\quad$ descriptive & {$[16]$} & {$[9],[35]$} & & 3 \\
\hline Total & 8 & 5 & 4 & 17 \\
\hline
\end{tabular}

Table 4: Outcome variables in the literature

\begin{tabular}{|l|l|l|}
\hline Outcome & Literature & Frequency \\
\hline Psychological & {$[\mathbf{2}],[\mathbf{5}],[\mathbf{9}],[\mathbf{1 0}],[\mathbf{2 8}],[\mathbf{3 5}],[\mathbf{4 0}],[\mathbf{4 2}],[\mathbf{4 4}],[\mathbf{4 6}],[49]$} & $\mathbf{1 2}$ \\
\hline - motivation & {$[\mathbf{2}],[\mathbf{1 0}],[\mathbf{2 8}],[\mathbf{3 3}],[\mathbf{4 0}],[\mathbf{4 2}],[\mathbf{4 4}],[\mathbf{4 6}]$} & \\
\hline - attitude & {$[\mathbf{2}],[\mathbf{2 8}],[\mathbf{4 6}]$} & \\
\hline - fun/enjoyment & {$[\mathbf{2}],[\mathbf{5}],[\mathbf{9}],[\mathbf{3 5}],[49]$} & \\
\hline Behavioral & $\begin{array}{l}{[\mathbf{2}],[\mathbf{5}],[\mathbf{9}],[\mathbf{1 0}],[\mathbf{1 6}],[22],[23],[\mathbf{2 7}],[\mathbf{2 8}],[30],[\mathbf{3 3}],[\mathbf{3 5}],[\mathbf{3 6}],[\mathbf{4 0}],} \\
{[\mathbf{4 2}],[\mathbf{4 4}],[\mathbf{4 5}],[\mathbf{4 6}],[\mathbf{2 3}],[49],[52],[\mathbf{5 3}],[55]}\end{array}$ \\
\hline \multicolumn{2}{|l}{ References in bold refer to studies in which empirical results about gamification have been reported. } \\
\hline
\end{tabular}

\section{Recommendations for gamifying crowdsourcing systems}

This review points to several recommendations for CS developers for using gamification. In the review we analyzed several types of studies that investigated the use of gamification in CS: 1) studies in which controlled experiments were conducted and detailed gamification design results thus provided (see Table 3 col. "Design studies"), as well as 2) studies reporting concrete implementations of gamified CS systems. In this section we describe what kinds of recommendations can be derived from the synthesis of literature on gamification in CS.

Points / scores: Nearly all of the examined systems use a metric (e.g points or scores) as a core element to reward measureable events in the human-system interaction. Due to this, we further analyzed the scoring mechanism used in the papers. Table 5 summarizes the findings clustered along the crowdsourcing types.
Rankings / leaderboards: The empirical findings indicate that rankings seem to be very effective to motivate certain users of a crowdsourcing community to contribute a lot by [36]. However, several studies show that the concrete design of a leaderboard has effects on the participation (in context of crowdprocessing [27, 36] and crowdrating [42, 46]). Based on these findings, [27] recommend short-term leaderboards, because "all-time" leaderboards can demotivate low-ranked participants and novices, for which the top seems impossible to reach. Studies by $[42,46]$ showed that long-term leaderboards can lead to demotivation and possible negative effects on the overall outcome of the crowdsourcing. The design of a leaderboard implementation seems therefore highly context dependent. [36] notes that many crowdsourcing approaches follow the "90-9-1" participation rule, implying that only $1 \%$ of the users perform almost all of the actions, and consequently, long-term leaderboards might also be suitable for many CS implementations. 
Table 5: Score design patterns for CS types

\begin{tabular}{|c|c|}
\hline Crowdprocessing & $\begin{array}{l}\text { Homogenous non-emergent tasks are easily enumerable. Therefore, most crowdprocessing } \\
\text { approaches reward the quantitative number of fulfilled tasks. This simple mechanism is usually } \\
\text { combined with further affordances like level systems and/or public leaderboards to achieve a } \\
\text { (self- or other-) competitive engagement }[27,36] \text {. However, leaderboards should be used } \\
\text { carefully, empirical studies on the use of leaderboards in crowdprocessing systems showed } \\
\text { positive and negative results }[27,36] \text {. }\end{array}$ \\
\hline & $\begin{array}{l}\text { In case of crowdrating the individual contributions represents a vote on a given topic. As an } \\
\text { aggregation of these votes a collective value emerges [15]. Therefore not only the quantity, but } \\
\text { also the quality is rewarded in most gamified crowdrating cases. Scoring mechanism, which set } \\
\text { the quality of a contribution in context of the emergent outcome (e.g. degree of agreement with } \\
\text { contributions of other crowdsourcees) are used to motivate users to emulate others and to "think } \\
\text { and act like the community". Similar to processing this mechanism is often combined with } \\
\text { leaderboards (see Table 1) or time pressure }[10,22,30] \text { to create a competition-based setting. }\end{array}$ \\
\hline & $\begin{array}{l}\text { Crowdsolving tasks strive for heterogeneous, non-emergent participations, which could be very } \\
\text { diverse and therefore hard to value by technical solutions. Based on the concrete problem, task } \\
\text { and implementation scoring mechanism that reward the quantitative participation or the quality } \\
\text { of the output could be suitable. This is very contextual and depends on the possibilities to } \\
\text { measure task fulfillment and/or task quality in a concrete use case. However, [5] provide first } \\
\text { empirical results about reward design in crowdsolving approaches. They showed in an } \\
\text { experiment that explicitly expressed gamification rewards before a crowdsolving task phase can } \\
\text { increase the quality of the CS work and the engagement of crowdsourcees. Furthermore, } \\
\text { engagement can be increased by implementing an open chance to achieve greater rewards } \\
\text { dependent upon the quality of their work. }\end{array}$ \\
\hline ating & $\begin{array}{l}\text { On a general level, crowdcreating systems aim at producing collaborative values by diverse and } \\
\text { creative contributions. In such systems, gamification can be used to motivate users towards, for } \\
\text { example, cooperation and creativity. Since only few studies could be found on gamification in } \\
\text { crowdcreation systems, no actual design patterns based on data can yet be described. However, } \\
\text { as the approach aims at gathering diverse contributions, implementing gamification in various } \\
\text { forms instead of, for example, merely points and badges and promoting cooperation rather than } \\
\text { competition could potentially be beneficial for reaching the common goal }\end{array}$ \\
\hline
\end{tabular}

Level systems: The empirical findings of [36] indicate that differences might exist between gamification designs with level systems that motivate by visualizing individual achievements and public participation rankings, which encourage workers to compare their effort with others. The results indicate that social achievements seem to be slightly more effective than personal level systems.

Manifold gamification approaches: Several examples use rich gamification designs with a diverse set of affordances (see Table 1). [42, 46] propose to mix several motivational affordances for different target groups to increase the overall outcome, which could be particularly important in crowdcreating and solving systems that benefit from the diversity of the participants. However, the experiment of [36] indicates that adding more motivational affordances does not always increase the motivation, especially in homogenous scenarios like crowdprocessing. Little knowledge is available so far, to explain effectiveness of affordances for specific user groups. Only [28] examined different target groups and showed that gamification does work for young and senior crowdsourcees, whereby competition-based gamification might be more effective for young rather than old participants. Several studies $[27,28,36,42]$ argue for the importance of intrinsic motivations like altruism or curiosity. Sustainable gamification designs should therefore be geared to user needs, suggesting more diversity than just points and leaderboards.

\section{Conclusions}

There has been a large variety of literature examining a wide array of different gamification implementations in all of the four types of CS initiatives. The literature seems to be unanimous; gamification seems to indeed work with majority of configurations and pairings with different CS types (crowdprocessing, -rating, -solving, and -creating). 
The empirical studies comparing gamified with nongamified approaches report an increase in engagement, output quality or other positive effects.

The literature, however, at this early stage is still quite scattered and not enough research has been conducted to draw clear conclusions as to which specific implementation would work better or worse in certain situations. It is clear that contextual factors and factors related to crowdsourcees play a role, but as to what extent and how is still unclear. Nevertheless, it is not an easy task to design gamification as also witnessed by the studies in the review. When designing an information system that attempts to affect human motivations and behavior, developers will inevitably end up with a complex design challenge.

What our study does show is that there are differences as to how gamification has been employed across different CS archetypes. Crowdsourcing initiatives that provide more monotonous tasks most commonly used mere points and other simpler gamification implementations, whereas in CS initiatives that seek for more diverse and creative contributions have employed gamification in more manifold ways employing a richer set of affordances (see Table 5). Regardless, points and leaderboards were clearly the most popular motivational affordances used in all four forms of crowdsourcing systems to create competition between the participants.

Several limitations should be noted both in the scope of this review as well as in the reviewed body of literature: 1) only few studies measured psychological aspects with rigorous measurement, 2) only few studies had carried out full experiments with control groups, 3) many studies clump multiple gamification mechanics in one and make it difficult to control from where the effect stems from, 4) gamification designs promoting cooperative behavior have been studied only in few cases, 5) due to the novelty of the phenomena the body of literature is limited and the topic has not yet been frequently addressed in high quality journals, and 6) the scope of this review was focused on studies investigating gamification particularly. However, it is possible that related research has been conducted also under other conceptual developments such as serious games, games-with-a-purpose, human-based computation games or persuasive technology. Conscious about these limitations, further studies on gamification (and crowdsourcing) should attempt to avoid them.

\section{References}

[1] L. von Ahn, "Human computation", In Proceedings of the 46th Annual Design Automation Conference DAC '09, 2009, San Francisco, IEEE, pp. 418-419.
[2] A. Bowser, D. Hansen, Y. He, et al., "Using gamification to inspire new citizen science volunteers", In Proceedings of the First International Conference on Gameful Design, Research, and Applications Gamification '13, 2013, Stratford, ACM, pp. 18-25. [3] M. Brenner, N. Mirza, and E. Izquierdo, "People recognition using gamified ambiguous feedback", In Proceedings of the First International Workshop on Gamification for Information Retrieval - GamifIR '14, 2014, Amsterdam, ACM, pp. 22-26.

[4] J. Chamberlain, "The annotation-validation (AV) model: rewarding contribution using retrospective agreement", In Proceedings of the First International Workshop on Gamification for Information Retrieval GamifIR '14, 2014, Amsterdam, ACM, pp. 12-16. [5] J. Choi, H. Choi, W. So, J. Lee, and J. You, "A Study about Designing Reward for Gamified Crowdsourcing System", In Proceedings of the 3rd International Conference, DUXU 2014, Held as Part of HCI International 2014, 2014, Heraklion, Springer International Publishing, pp. 678-687.

[6] S. Cooper, F. Khatib, A. Treuille, et al., "Predicting protein structures with a multiplayer online game.", Nature, 466(7307), 2010, pp. 756-760.

[7] E.L. Deci, "Effects of externally mediated rewards on intrinsic motivation", Journal of Personality and Social Psychology, 18(1), 1971, pp. 105-115.

[8] E.L. Deci, R. Koestner, and R.M. Ryan, "A metaanalytic review of experiments examining the effects of extrinsic rewards on intrinsic motivation", Psychological bulletin, 125(6), 1999, pp. 627-668. [9] A. Dumitrache, L. Aroyo, C. Welty, R.-J. Sips, and A. Levas, "Dr. Detective: combining gamification techniques and crowdsourcing to create a gold standard for the medical domain", In Proceedings of the 1st International Workshop on Crowdsourcing the Semantic Web (Crowd Sem2013), 2013, Sydney, pp. 16-31.

[10] C. Eickhoff, C.G. Harris, A.P. de Vries, and P. Srinivasan, "Quality through flow and immersion", In Proceedings of the 35th international ACM SIGIR conference - SIGIR '12, 2012, Portland, ACM Press, pp. 871-880.

[11] P.D. Ellis, The essential guide to effect sizes: Statistical power, meta-analysis, and the interpretation of research results, Cambridge University Press, Cambridge, 2010.

[12] Elsevier B.V., "Scopus", http://www.scopus.com/, June 15, 2015.

[13] E. Estellés-Arolas and F. Gonzalez-Ladron-deGuevara, "Towards an integrated crowdsourcing definition", Journal of Information Science, 38(2), 2012, pp. 189-200.

[14] Gartner, "Gartner says by 2015, more than 50 percent of organizations that manage innovation 
processes will gamify those processes.", http://www. gartner. com/it/page.jsp?id=1629214, April 11, 2011. [15] D. Geiger and M. Schader, "Personalized task recommendation in crowdsourcing information systems - Current state of the art", Decision Support Systems, 65, 2014, pp. 3-16.

[16] J. Goncalves, S. Hosio, D. Ferreira, and V. Kostakos, "Game of words: tagging places through crowdsourcing on public displays", In Proceedings of the 2014 conference on Designing interactive systems DIS '14, 2014, Vancouver, ACM, pp. 705-714. [17] J. Hamari, "Transforming homo economicus into homo ludens: A field experiment on gamification in a utilitarian peer-to-peer trading service", Electronic Commerce Research and Applications, 12(4), 2013, pp. 236-245.

[18] J. Hamari, K. Huotari, and J. Tolvanen, "Gamification and economics", In Walz, S.P. and S. Deterding, eds., The Gameful World: Approaches, Issues, Applications, MIT Press, Cambridge, 2015, pp. 139-161.

[19] J. Hamari and J. Koivisto, "Why do people use gamification services?", International Journal of Information Management, 35(4), 2015, pp. 419-431. [20] J. Hamari, J. Koivisto, and H. Sarsa, "Does Gamification Work? -- A Literature Review of Empirical Studies on Gamification", In Proceedings of the 47th Hawaii International Conference on System Sciences - HICSS, 2014, Waikoloa, IEEE, pp. 30253034.

[21] J. Hamari, M. Sjöklint, and A. Ukkonen, "The sharing economy: Why people participate in collaborative consumption", Journal of the Association for Information Science and Technology, forthcoming, 2015.

[22] C.G. Harris, "The Beauty Contest Revisited: Measuring Consensus Rankings of Relevance using a Game", In Proceedings of the First International Workshop on Gamification for Information Retrieval GamifIR '14, 2014, Amsterdam, ACM, pp. 17-21.

[23] J. He, M. Bron, and L. Azzopardi, "Studying User Browsing Behavior Through Gamified Search Tasks", In Proceedings of the First International Workshop on Gamification for Information Retrieval - GamifIR '14, 2014, Amsterdam, ACM, pp. 49-52.

[24] J. Howe, "The Rise of Crowdsourcing", Wired, 14(6), 2006.

[25] K. Huotari and J. Hamari, "Defining gamification", In Proceeding of the 16th International Academic MindTrek Conference on - MindTrek '12, 2012, Tampere, ACM Press, pp. 17.

[26] IEEE, "Everyone's a Gamer - IEEE Experts Predict Gaming Will Be Integrated Into More than 85 Percent of Daily Tasks by 2020", http://www.ieee.org/ about/news/2014/25_feb_2014.html, April 14, 2014.
[27] P.G. Ipeirotis and E. Gabrilovich, "Quizz: Targeted Crowdsourcing with a Billion (Potential) Users", In Proceedings of the 23rd international conference on World wide web - WWW'14, 2014, Seoul, ACM, pp. 143-154.

[28] T. Itoko, S. Arita, M. Kobayashi, and H. Takagi, "Involving senior workers in crowdsourced proofreading", In Proceedings of the 8th International Conference, UAHCI 2014, Held as Part of HCI International 2014, 2014, Heraklion, Springer International Publishing, pp. 106-117.

[29] J.H. Jung, C. Schneider, and J. Valacich, "Enhancing the Motivational Affordance of Information Systems: The Effects of Real-Time Performance Feedback and Goal Setting in Group Collaboration Environments", Management Science, 56(4), 2010, pp. 724-742.

[30] H. Kacorri, K. Shinkawa, and S. Saito, "Introducing game elements in crowdsourced video captioning by non-experts", In Proceedings of the 11th Web for All Conference on - W4A '14, 2014, Seoul, ACM, pp. 1-4.

[31] B. Kanefsky, N.G. Barlow, and V.C. Gulick, "Can Distributed Volunteers Accomplish Massive Data Analysis Tasks?", In Proceedings of the 32th Annual Lunar and Planetary Science Conference, 2001, Houston.

[32] N. Kaufmann, T. Schulze, and D. Veit, "More than fun and money. Worker Motivation in Crowdsourcing - A Study on Mechanical Turk.", In Proceedings of the 17th Americas Conference on Information Systems - Amcis, 2011, Detroit, pp. 1-11. [33] R. Kawajiri, M. Shimosaka, and H. Kahima, "Steered crowdsensing: Incentive Design towards Quality-Oriented Place-Centric Crowdsensing", In Proceedings of the 2014 ACM International Joint Conference on Pervasive and Ubiquitous Computing UbiComp '14, 2014, Seattle, ACM, pp. 691-701. [34] J. Koivisto and J. Hamari, "Demographic differences in perceived benefits from gamification", Computers in Human Behavior, 35, 2014, pp. 179188.

[35] J.J. Lee, P. Ceyhan, W. Jordan-Cooley, and W. Sung, "GREENIFY: A Real-World Action Game for Climate Change Education", Simulation \& Gaming, 44(2-3), 2013, pp. 349-365.

[36] T.Y. Lee, C. Dugan, W. Geyer, et al., "Experiments on motivational feedback for crowdsourced workers", In Proceedings of the 7th International Conference on Weblogs and Social Media - ICWSM 2013, 2013, AAAI Press, pp. 341350.

[37] J.M. Leimeister, "Collective Intelligence", Business \& Information Systems Engineering, 2(4), 2010, pp. 245-248. 
[38] C.J. Lintott, K. Schawinski, A. Slosar, et al., "Galaxy Zoo: Morphologies derived from visual inspection of galaxies from the Sloan Digital Sky Survey", Monthly Notices of the Royal Astronomical Society, 389(3), 2008, pp. 1179-1189.

[39] Y. Liu, T. Alexandrova, and T. Nakajima, "Gamifying intelligent environments", In Proceedings of the 2011 international ACM workshop on Ubiquitous meta user interfaces - Ubi-MUI '11, 2011, Scottsdale, ACM, pp. 7-12.

[40] Y. Liu, T. Alexandrova, T. Nakajima, and V. Lehdonvirta, "Mobile Image Search via Local Crowd: a User Study", In Proceedings of the 17th International Conference on Embedded and Real-Time Computing Systems and Applications (RTCSA 2011), 2011, Los Alamitos, IEEE Computer Society, pp. 109-112. [41] A.D. Mason, G. Michalakidis, and P.J. Krause, "Tiger Nation: Empowering citizen scientists", In Proceeding of the 6th IEEE International Conference on Digital Ecosystems and Technologies (DEST), 2012, IEEE, pp. 1-5.

[42] E. Massung, D. Coyle, K.F. Cater, M. Jay, and C. Preist, "Using crowdsourcing to support proenvironmental community activism", In Proceedings of the SIGCHI Conference on Human Factors in Computing Systems - CHI '13, 2013, Paris, pp. 371380 .

[43] Y. Nagai, A. Hiyama, T. Miura, and M. Hirose, "T-echo: Promoting intergenerational communication through gamified social mentoring", In Proceedings of the 8th International Conference - UAHCI 2014, Held as Part of HCI International 2014, 2014, Heraklion, Springer, pp. 582-589.

[44] T. Nose and R. Hishiyama, "Analysis of selftagging during conversational chat in multilingual gaming simulation", In 2nd International Conference on Future Generation Communication Technologies, FGCT 2013, 2013, London, IEEE, pp. 81-86. [45] D. Pothineni, P. Mishra, A. Rasheed, and D. Sundararajan, "Incentive Design to Mould Online Behavior: A Game Mechanics Perspective", In Proceedings of the First International Workshop on Gamification for Information Retrieval - GamifIR '14, 2014, Amsterdam, ACM, pp. 27-32.

[46] C. Preist, E. Massung, and D. Coyle, "Competing or aiming to be average?: Normification as a means of engaging digital volunteers", In Proceedings 17th ACM Conference on Computer Supported Cooperative Work and Social Computing, 2014, Baltimore, ACM, pp. 1222-1233.

[47] S. Saito, T. Watanabe, M. Kobayashi, and H. Takagi, "Skill development framework for microtasking", In Proceedings of the 8th International Conference, UAHCI 2014, Held as Part of HCI
International 2014, 2014, Heraklion, Springer, pp. 400-409.

[48] M. Sakamoto and T. Nakajima, "Gamifying social media to encourage social activities with digitalphysical hybrid role-playing", In Proceeding of the 6th International Conference, SCSM 2014, Held as Part of HCI International 2014, 2014, Heraklion, Springer, pp. 581-591.

[49] L.Y. Sheng, "Modelling learning from Ingress (Google's augmented reality social game)", In Proceedings of the 2013 IEEE 63rd Annual Conference International Council for Education Media, ICEM, 2013, Singapore, IEEE, pp. 1-8.

[50] T. Straub, H. Gimpel, F. Teschner, and C. Weinhardt, "How (not) to Incent Crowd Workers", Business \& Information Systems Engineering, 57(3), 2015, pp. 167-179.

[51] J. Surowiecki, The wisdom of crowds, Anchor Books, New York, 2005.

[52] A. Uzun, L. Lehmann, T. Geismar, and A. Küpper, "Turning the OpenMobileNetwork into a live crowdsourcing platform for semantic context-aware services", In Proceedings of the 9th International Conference on Semantic Systems - I-SEMANTICS '13, 2013, Graz, ACM, pp. 89-96.

[53] B. Vasilescu, A. Serebrenik, P. Devanbu, and V. Filkov, "How social Q\&A sites are changing knowledge sharing in open source software communities", In Proceedings of the 17th ACM conference on Computer supported cooperative work \& social computing - CSCW '14, 2014, Baltimore, ACM, pp. 342-354.

[54] J. Webster and R.T. Watson, "Analyzing the Past to Prepare for the Future: Writing a Literature Review", MIS Quarterly, 26(2), 2002, pp. xiii-xxiii. [55] D. Yakushin and J. Lee, "Cooperative robot software development through the internet", In Proceedings of the 2014 IEEE/SICE International Symposium on System Integration (SII), 2014, Tokyo, IEEE, pp. 577-582.

[56] P. Zhang, "Motivational Affordances: Reasons for ICT Design and Use", Communications of the ACM, 51(11), 2008, pp. 145-147.

[57] Y. Zhao and Q. Zhu, "Evaluation on crowdsourcing research: Current status and future direction", Information Systems Frontiers, 16(3), 2014, pp. 417-434.

[58] Y. Zhao and Q. Zhu, "Effects of extrinsic and intrinsic motivation on participation in crowdsourcing contest", Online Information Review, 38(7), 2014, pp. 896-917.

[59] H. Zheng, D. Li, and W. Hou, "Task Design, Motivation, and Participation in Crowdsourcing Contests", International Journal of Electronic Commerce, 15(4), 2011, pp. 57-88. 